\section{Leave biofilm dead in the water}

For almost ten years, CleanCert has been providing dental practices and laboratories with effective and 'easy to use' infection control solutions.

CleanCert+ biofilm cleaner, for example, is a revolutionary one-stage waterline cleaner that is independently proven to be efficacious, as well as safe and simple to use. Used just once a week for five minutes (using reverse osmosis or distilled water the rest of the time) and priced at only $£ 41$ for a six-month supply for one dental chair, it offers incredible value and unrivalled safety (MSDS).

LabCert, meanwhile, is a powerful and highly effective ready-to-use disinfectant, which can be employed on all materials and appliances that are transferred between dental practices and laboratories, ensuring the wellbeing of staff and patients alike in all premises.

For further information on how to simplify your infection control HTM 01-05 'best practice' compliance with the full range of proven, innovative dental infection control and water purification products available from CleanCert, visit cleancert.co.uk or call 08443511115.

\section{Revitalise your patient dental chairs}

Meditelle-Dental provides a professional onsite re-covering service to rejuvenate your dental patient chairs at your surgery.

This onsite service covers the whole of the UK and specialises in the reupholstering of most makes and models of patient dental chairs. All procedures are carried out at your premises ensuring your equipment is not out of service for any length of time, minimising disruption and downtime. Flexible appointments and early time slots prior to opening are offered to work around your practice's schedule.

Meditelle's re-covering service will keep your patient chairs hygienic and ensure that the safety of your equipment is maintained. Re-upholstering may be all that's required if you have any scuffs, slits

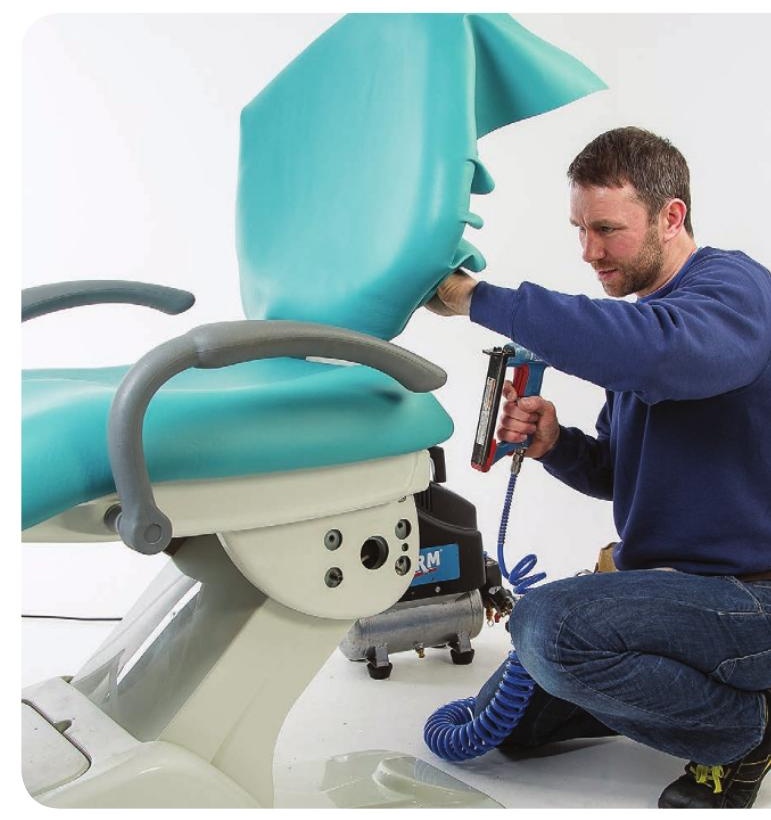
or tears in your dental chair vinyl that could present an infection control hazard.

Meditelle uses hydrophobic, anti-microbial medical grade vinyls which are MRSA and E-coli resistant and offered in a wide range of colours.

Offered in conjunction with this specialist service are the additional options of having a made-to-measure foot guard fitted and your operator stools re-covered to match your revitalised dental chair.

Obtaining a no-obligation quotation couldn't be easier, just email a full image of your chair front and back to Meditelle along with your postcode.

Telephone 0121332 1850, email enquiry@meditelle.co.uk or visit www.meditelle-dental.co.uk.

\title{
Another legend is born
}

The Straumann Bone Level Tapered (BLT) $\varnothing 2.9 \mathrm{~mm}$ implant is the implant solution for narrow interdental spaces or ridges. The smallest in the Bone Level Tapered range, it completes the portfolio for narrow space indications and integrates seamlessly with the Straumann Dental Implant System.

Benefitting from the clinically-proven BLT design, it has a parallel body with full-depth thread and three cutting notches for selfcutting in underprepared sites. Its apically tapered design delivers excellent primary stability in soft bone and fresh extraction sockets as it adequately compresses the underprepared osteotomy and allows the clinician to effectively master the patient's anatomy, even when limited, and protects the anatomical structure with its round tip.

All Straumann BLT implants also possess the powerful combination of Roxolid, SLActive, Bone Control Design, CrossFit connection and prosthetic diversity.
Roxolid has enabled Straumann to create smaller implants than before, thus reducing invasiveness, by preserving bone and minimising the amount of bone grafting required. Roxolid is a high performance alloy, specifically designed to offer higher strength than pure titanium, making these smaller implants possible. Smaller implants increase the range of treatment options available to the clinician and patient acceptance will be higher when less invasive treatment options are offered.

The SLActive surface allows fast and predictable osseointegration, enabling safer and faster treatment and higher treatment predictability in challenging protocols. Clinically proven to provide safer and faster treatment in 3-4 weeks for all indications, it increases safety during the early healing phase and leads to a superior structural and functional connection between the implant and vital bone.

Bone Control Design optimises crestal bone preservation while the CrossFit connections simplify handling and increase confidence in component positioning, ensuring precision against rotation and long-term flexibility.

Prosthetic diversity is ensured by compatibility with the full range of Straumann prosthetic components, including the unique oval design for optimal soft tissue management in smaller spaces.

The BLT Ø $2.9 \mathrm{~mm}$ implant is indicated for single-tooth restorations of the central and lateral incisors in the mandible, and lateral incisors in the maxilla.

Coming soon... single use instruments will allow you to place the BLT Ø $2.9 \mathrm{~mm}$ implant with sterile, disposable burs, drills, taps and profilers, ensuring your instruments are always sharp and minimising the cost and inconvenience of sterilisation.

For more information on the range of Straumann Bone Level Tapered Implant solutions, contact Straumann on 01293651230 or visit straumann.co.uk. 